\title{
A Best-Match Algorithm for Broad-Coverage Example-Based Disambiguation
}

\author{
Naohiko URAMO'TO \\ IBM Ressearch, Tolyo Rescarch Laboratory \\ 1623-14 Simotsuruma, Yamato-shi, Kanagawa-ken 242 Japan \\ uramoto@itrl.vnet.ibrn.com
}

\begin{abstract}
To improve the coverage of example-bases, two methods are introduced into the best-match algorithm. The first is for acquiring conjunctive relationships from corpora, as measures of word similarity that can be used in addition to thesauruses. The Second, used when a word does not appear in an example-base or a thesaurus, is for inferring links to words in the example-base by comparing the usage of the word in the text and that of works in the example-base.
\end{abstract}

\section{Introduction}

Improvement of coverage in practical clomains is one of the most important issues in the area of examplebased systems. The examplo-based approach [6] has become a common technique for natural language processing applications such as machine translation and disambiguation (e.g. $[5,10]$ ). However, fow existing systems can cover a practical domain or handle a broad range of phenomena.

The most scrious obstacle to robust examplebased systems is the coverage of example-bases. It is an open question how many examples are required for disambiguating sentences in a specific domain.

The Sentence Analyzer (SENA) was developed in order to resolve attachment, word-sense, and conjunctive ambiguities by using constraints and example-based preferences [11]. It has about 57,000 disambiguated head-modificr relationships and about 300,000 synonyms and is-a binaryrelationships. Even so, lack of examples (no relevant examples) accounted for $46.1 \%$ of failures in a experiment with SENA [12].

Previously, it was belicved to be easier to collect examples than to develop rules for resolving ambiguities. However, the coverage of each example is much more local than a rule, and therefore a huge number of examples is required in order to resolve realistic problems. There has been some corpusbased research on how to arcquire large-scale knowledge antomatically in order to cover the domain to be disambiguated, but there are still major problems to be overcone.
First, semantic kuowledge such as word-sense cannot be extracted by autonatic corpus-based knowledge acquisition. The exanple-base in SENA is developed by using a bootstrapping method. However, the results of word-sense disambiguation must be checked by a human, and word-senses are tagged to only about a half of all the examples, since the task is very time-consuming.

A second difficulty in the example-based approach is the algorithm itself, namely, the bestmatch algorithm, which was used in earlier systems built around a thesaurus that consisted of a hierarchy of is-a or synonym relationships between words (word-senses).

This paper proposes two methods for improving the coverage of example-bases. The selected domain is that of sentences in computer manuals. First, knowledge that represents a type of sinnilarity other than synonym or is-a relationships is acquired. As one neasumement of the similarity, interchangeability between words can be used. In this paper, two types of the relationship reflect such interchangeability. First, the elements of coordinated structures are good clues to the interchangeability of words. Words can be extracted easily from a domain-specific corpus, and therefore the examplebase can be adapted to the specific domain by using the domain-specific relationships.

If there are no examples and relations in the thesaurus, the example-base gives no information for disambignation. However, the text to be disambignated provides useful knowledge for this purpose $[7,3]$. 'The relationships between words in the example-base and an muknown word can be guessed by comparing that word's nsage in extracted exanples and in the text.

\section{A Best-Match Algorithm}

In this section, conventional algorithms for example-based disambiguation, and their associated problems, are brielly introduced. The algorithms of most example-lased systens consist of the fol lowing three steps':

\footnotetext{
In some systems, the exact-match and the best-match are merged.
} 


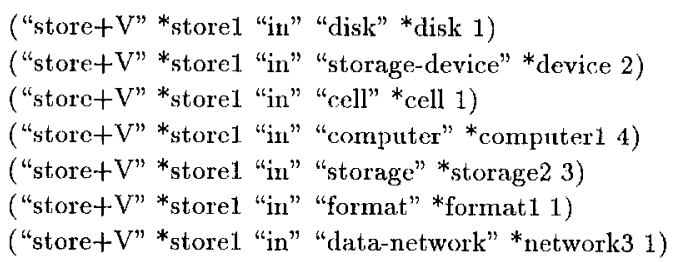

Fig. 1: Examples for R1

("program $+N$ " * prog1 "in" "profile $+N$ " *profile 5) ("program+N" *prog1 "in" "clata-storage+N" *storage3 1)

("program $+N$ " * prog 1 "in" "publication $+N " *$ publication 12$)$

("program+N" *prog1 "in" "form $+N$ " *form1 2)

("program+N" *prog2 "in" "group $+N " *$ group1 1)

Fig. 2: Examples for R2

1. Searching for examples

2. Exact matching

3. Best matching with a thesaurus

Suppose the prepositional phase attachment ambiguity in $\mathrm{S} 1$ is resolved by using these steps.

(S1) A managed AS/400 system can store a new program in the repository.

There are two candidates for the attachment of the prepositional phrase "in the repository." 'They are represented by the following head-modifier relationships:

(R1) ("store+V" (PP "in") "repository+N")

(R2) ("program+N" (PP "in") "repository+N")

In R1 the noun "repository" modifies the verb "store" with "in," while in R2, it modifies the noun "program."

First, SENA searches for examples whose heads match the candidate. Figures 1 and 2 show the relevant examples for $\mathrm{R} 1$ and $\mathrm{R} 2$. They lepresent the head-modifier relationships, including wordsenses, a relation label between the word-senses, (c.g. "in"), and a frequency.

If a relationship identical to either of the candidates $R 1$ and $R 2$ is found, a high similarity is attached to the candidate and the example (cxact matching).

Word-sense ambiguities are resolved by using the same framework [12]. In this case, each candidate represent each word sense. For exarnple, the word-sense ${ }^{*}$ store 1 is preferred among the examples shown in Fig. 1.

If no examples are obtained by the exactmatching process, the system executes the bestmatching process, which is the most important mechanism in the example-based approach. For the comparison, synonym or is-a relationships described in a thesaurus are used. For example, if synonym relations are found between "lepository" and "disk" in the first example for the R1, a similarity whoso value is smaller than that for exact matching is given to the examples. 'The most preferable candidate is selected by comparing all examples in Fig. 1 and computing the total similarity value for each candidate. If multiple candidates have the same similarity values, the frequency of the example and some heuristics (for example, innermost attachment is preferred) are used to weight the similarities.

Experience with SENA reveals two problems that prevent an improvement in the performance of the best-matching algorithm. First, the approach is strongly dependent on the thesaurus. Many systems calculate the similarity or preference mainly or entircly by using the hicrarchy of the thesaurus. However, these relationships indicate only a certain kind of similarity between words. To improve the coverage of the example-base, other additional types of knowledge are required, as will be discussed in the following sections.

Another problem is the existence of unknown words; that is, words that are described in the system dictionary but do not appear in the examplebase or the thesaurus. In SENA, the New Collins Thesaurus [1] is used to disambiguate sentences in computer manuals. Many unknown words appear, especially nouns, since the thesaurus is for the general domain. Therefore, a mechanism for handling the unknown words is required. This is covered in Chapter 4.

\section{Knowledge Acquisition for Robust Best-Matching}

As described in the previous section, the bestmatching algorithm is a basic element of examplebased disambiguation, but is strongly dependent on the thesaurus. Nirenburg [8] discusses the type of knowledge needed for the matching; in his method, morphological information and antonyms are used in addition to synonym and is-a relationships. This section discusses the acquisition of knowledge from other aspects for a broad-coverage best-match algorithm.

\subsection{Acquisition of Conjunctive Rela- tionships from Corpora}

The New Collins Thesaurus, which is used in SENA as a source of synonym or is-a relationships, gives the following synonyms of "store":

store:

accumulate, deposit, garner, hoard, keep, etc.

In our example-base, there are fow examples for any of the words except "keep," since the examplebase was developed mainly to resolve sentences in technical documents such as computer manuals. When the domain is changed, the vocabulary and 
the usage of words also change. Fven a generaldomain thesaurus sometimes does not suit a specific domain. Moreover, development of a domainspecific thesaurns is a time-consuming task.

The use of synonym or is-a relationships suggests the hypothesis that from the viewpoint of the example-based approach, a word in a sentence can be replaced by its synonyms or taxonyms. That is, it supports the existence of the (virtual) example S1' when "store" and "keep" have a synonym relationship.

(S1') A managed AS/400 system can keep a now program in the repository.

Interchangeability is an important condition for calculating similarity or preferences between words. Our claim is that if words are interchangeable in sentences, they should have strong similarity.

In this paper, conjunctive relationships, which are common in technical documents, are proposed as relationships that satisfy the condition of interchangeability. Sentences in which the word "store" is used as an element of coordinater structure can be extracted from computer manuals, as following examples show:

(1) The service retrieves, formats, and stures a message for the uscr.

(2) Delete the identifier being stored or modified from the table.

(3) This EXEC verifics and stores the language defanlts in your file.

(4) You use the function to add, store, retrieve, and update information about docunents.

From the sentences, the following words that are interchangeable with "store" are accluired:

store: retrieve, format, modify, verify, add, update.

Often the words share case-pattens, which is a useful characteristic for determining interchange-. ability. Another reason we use conjunctive relationships is that they can be extracted semiantomatically from untagged or tagged corpora by using a simple patten-matching nethod. We extracted about 700 conjunctive relationships from nntagged computer mannals by pattern matching. The relationships include varions types of knowledge, such as 1 (a) antonyms (e.g. "private" and "public"), (b) sequences of actions (c.g. "load" and "edit"), (c) (weak) synonyns (c.g. "program" and "service"), and (d) part-of relationships (c.g. "tape" and "device"). Another merit of conjunctive relationships is that they reflect domain-specific relations.

\subsection{Acquisition from Text to Be Dis- ambiguated}

If there are no exanples of a word to be disambignated, and the word does not appear in the thesaurus, no relationships are acquired.
The existence of words that are nuknown to the example-base and the thesaurus is incvitable when one is dealing with the disanubignation of sentences in practical domains. Computer mantuals, for example, contain many special nouns such as natues of commands and products, but, there are no thesauruses for such highly domain-specifis: words.

One way of resolving the problem is to use the text to be processed as the most domain-specific example-base. This idea is supported by the fact that most word to-word dependencies including the nuknown words appear many tines in the sano text. Nasukawa [7] developed the Discontse Analyzer (I)LANA), which resolves ambiguities in a text by dynamically referting to contextual information. Kinoshita et al. [3] also proposed a method for machine translation by parsing a complete text in advance aud using it as an example-base. Howeyer, neither system works for mnknown words, since both use only apendencies that appear explicitly in the text.

\section{An Algorithm to Search for Unknown Words}

We first give an culanced best-match algorithm for disambignation. The steps given in Chaptex 2 are noolified ats follows:

1. Soarching for examples

2. Fxast matching

3. Best matching with a thesaurus and conjunctive relationships

4. Unknown-word-malching using a context-basse 'The outline of the the algorithm is as follows: Sentences in the text to be processed are parsed in advance, and the parse trees are stored as a contextbase. 'l'he context-base can include anbignons word-to-word dependencies, since no disambignation process is executed. Using an example-base and the context-luase, the sentences in the text are disambiguated sequentially. If an ambignons worl does not appeas in an example-base or in the thesaurus, an unknown word search is executed (otherwise, the conventional best-match process is executad.) The nuknown-word-matching process includes the following steps:

L. l'he depondencies that inclucle the unknown word are extracted from the context-base.

2. A candidate set of words that is interchangeable with the unknown word is searched for in the example-base by using the context dependency.

3. The candidate set acculired in step 2 is compared with the examples extracted for each candidate of interpretation. A preference valwe is calculated by using the sets, and the most prefered interpretation is selected. 
Let us see how the algorithm resolves the attachment ambiguity in sentence $\mathrm{S} 1$ from Chapter 2, which is taken from a text (manual) for the AS/400 system.

(S1) A managed AS/400 system can store a new program in the repository.

The text that contains $S 1$ is parsed in advance, and stored in the context-base. The results of the example search are shown in Fig. 1. There are two candidate relationships for the attachment of the prepositional phrase "in the repository".

(R1) ("store+V" (PP "in") "repository+N")

(R2) ( "program+N" (PP "in") "repository $+N$ ")

The noun "repository" does not appear in the example-base or thesaurus, and therefore no information for the attachment is acquired.

Consequently, the word-to-word dependencies that contain "repository" are searched for in the context-base. The following sentences appear before or after $\mathrm{S} 1$ in the text:

(CB1) The repository can hold objects that are ready to be sent or that have been received from another user library.

(CB2) A distribution catalog entry exists for each object in the distribution repository. (CB3) A data object can be loaded into the distribution repository from an AS/400 library. (CB4) The object type of the object specified must match the information in the distribution repository.

From the sentences, the head-modifier relationships that contain the unknown word "repository" are listed. These relationships are called the context dependency for the word. The context dependency of "repository" is as follows:

(D1) ("hold+V" (subj) "repository+N"): 1

(D2) ("cxist+V" (PP "in") "repository+N") : 0.5

(D3) ("object+N" (PP "in") "repository+N"): 0,5

(D4) ("load+V" (PP "into") "repository+N"): 1

(D5) ("information+N" (PP "in") "repository+N") : 0.5

(D6) ("match+V" (PP "into") "repository+N"): 0.5

The last number in each relation is the certainty factor (CF) of the relationship. The value is $1 /$ (the number of candidates for the resolving ambiguity). For example, the attachment of "repository" in CB2 has two candidates, D2 and D3. Therefore, the certainty factors for D2 and D3 are $1 / 2$.

For each dependency, candidate words (CB) in the context-base are searched for in the examplebase. The words in the set can be considered as substitutable synonyms of the unknown word. For example, the WORDs that satisfy the relationship ("hold +V" (subj) WORD+N) in the case of D1 are searched for. The following are candiclate words in the context-base for the word "repository."

$\mathrm{CB} 1=\{\mathrm{I}$, user, cradle, rock $\}$ (for D1)

$\mathrm{CB} 2=$ storage, transient data $\}$ (for D2)

$\mathrm{CB3}=\{$ condition, format, path, 1916, technique, control area\} (for D3)

$\mathrm{CB} 4=\{$ system 38, farcility $\}$ (for D4)

$\mathrm{CB} 5=\{$ recold $\}$ (for D5)

$\mathrm{CB} 6=\{\}($ for D6)

The total set of candidate words (CB) of the "repository" is an union of CB1 through CB6. The set is compared with the extracted examples for each attachment candiclate (Fig. 1). The words in the examples are candidate words in the examplebase. By intersecting the candidate words in the context-base and the example-base, word that are interchangeable with the unknown word can be extracted. The intersections of each set are as follows:

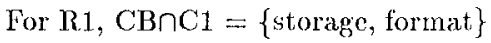

For $\mathrm{R} 2, \mathrm{CB \cap C} 2=\{\}$

This result means that "storage" and "format" have the same usage (or are interchangeable) in the text. The preference value $P(R)$ for the candidate $\mathrm{R}$ with the interchangeable word $w$ is calculated by the formula:

$$
P(R)=\sum_{w}(C F) \times(\text { frequency })
$$

In this case, $\mathrm{P}(\mathrm{R} 1)=0.5 \times 1+0.5 \times 1=1.0$, and $P(R 2)=0$ (supposing that the frequency of the words is 1). As a result, $R 1$ is preferred to $R 2$.

If both sets of candidates are cinpty, the numbers of extracted examples are compared (this is called Heuristic-1). If there are no related words in this case, R1 is preferred to R2 (see Fig. 1). This heuristic indicates that "in" is preferred after "store," irrespective of the head word of the prepositional phrase.

\section{Experimental Results}

\subsection{Example-Base and Thesaurus}

An example-base for disambiguation of sentences in computer manuals is now being developed. Table 1 shows its current size. The sentences are extracted from examples in the Longman Dictionary of Contemporary English [9] and definitions in tlıe IBM Dictionary of Computing [2]. Synonym and is-a relationships are extracted from the New Collins Thesaurus [1] and Webster's Seventh New Collegiate Dictionary [4].

Our example-hase is a set of head-modifier binary dependencies with relations between word, such as (subject), (object), and (PP "in"). It was developed by a bootstrapping method with human correction. In SENA, the examplo-base is used to resolve three types of ambiguity: attachment, word-sense, and coordination. The level of knowledge depends on the type of ambiguity. 
Table 1: Size of the Example-Base and Thesaurus

\begin{tabular}{|c|c|}
\hline \multicolumn{2}{|l|}{ Example-Base } \\
\hline Examples & $\begin{array}{l}57,170 \text { binary relationships } \\
\text { (in } 9,500 \text { sentences) }\end{array}$ \\
\hline Distinct words & 8,602 \\
\hline \multicolumn{2}{|l|}{ Thesaurus } \\
\hline Synonyms & $\begin{array}{r}283,211 \text { binary relationships } \\
\text { (11,006 entries) }\end{array}$ \\
\hline Is-a relations & 6,353 binary relationships \\
\hline
\end{tabular}

\begin{tabular}{|l} 
Success with unknown word matching \\
$52.4(\%)$
\end{tabular}

\begin{tabular}{|l|l|}
\hline Success with Heuristic-1 & $20.0(\%)$ \\
\hline
\end{tabular}

Failure $27.6(\%)$

Fig. 3: Result of disambiguation

To resolve semantic ambiguities, the examples should be disambiguated semantically. On the other hand, structural dependencies can be extracted from raw or tagged corpora by using simple rules or patterns. In our approach, multilevel descriptions of examples are allowed: one example may provide both structural and word-sense information, while another may provide only structural dependencies. Word-senses are ardded to a half of the sentences in example-base.

\subsection{Experiment}

We did a small experiment on disambiguation of prepositional phrase attachment. F'irst, we prepared 105 ambiguons test data randomly from 3,000 sentences in a computer manual. The format of the data was as follows:

verb noun prep unknown-noun

None of these data can be disambiguated by using the conventional best-matching algorithm, since noun2 does not appear in the example-base or thesaurus. Conjunctive relationships, described in Chapter 3 , are used with the example-base and the thesaurus.

The results of the disambiguation are shown in Fig. 3. We were able to disambiguate $52.4 \%$ of the test data by using unknown-word-matching. By using Heuristic-1 in addition, we obtained a $72.4 \%$ success rate for unknown words.

One cause of failure is imbalance among examples. The number of examples for frequent verbs is larger than the number of examples for frequent nouns. As a result, verb attachment tends to be preferred. $^{2}$ Another cause of failure is the number of context dependencies. In the experiment, at most the nearest eight sentences were used; the optimum number is still an open question.

\footnotetext{
${ }^{2}$ We did not use other heuristies such as preference for inner attachment.
}

\section{Conclusion}

Methods for improving the coverage of examplebases were proposed in order to allow the realization of broad-coverage example-based systems. We are evaluating our approach with larger amounts of data. For future progress, the following issues must be discussed:

1. In this paper, conjunctive relationships were nsed as knowledge with the best-match algorithm, in addition to a thesaurus. However, various types of knowledge will be required on a large scale for a more robust system. Automatic or semi-automatic accuisition, using corpus-based methods, is also needed.

2. If there are many unknown words in an ambiguity, unknown-word matching will not work well. In acldition to scaling up the examplebase and the thesaurus, we should develop a more robust algorithm.

\section{References}

[1] Collins. The New Colling Thesaurus. Collins Publishers, Glasgow, 1984.

[2] IBM Corporation. IBM Dictionary of Computing, volume SC20-1699-07. IBM Corporation, 1988.

[3] S. Kinoshita, M. Shinazn, and H. Hirakawa. "Better Translation with Knowledge Extracted from Source Text". In Proceedings of TMI-93, pages 240-252, 1993.

[4] Merrians. Webster's Seventh New Collegiate Dictionary. G.\& C. Merriam, Springfield, Massachusetts, 1963.

[5] K. Nagao. "Dependency Analyzer: A KnowledgeBased Approach to Structural Disambignation". In Procedings of COIING-90, pages 282-287, 1990.

[6] M. Nagao. "A Framework of a Mechanical Trauslation between Japanese and English by Analogy Principle". In A. Wlithorn and R. Banerji, editors, Artificial and Human Intelligence. NATO, 1984.

[7] T. Nasukawa. "Discourse Constraint in Computer Manuals". In Proceedings of 'TMI-93, pages 183 $194,1993$.

[8] S. Nirenburg, C. Domashnev, and D. I. Grannes. "Two Approaches to Matching in Example-Based Machine Trunslation". In Procecdings of TMI-99, pages $47-57,1993$.

[9] P. P'octer. Longman Dictionary of Contemporary Innglish. Longman Group Limited, Harlow and London, England, 1978.

[10] S. Sato and M. Nagao. "Towards Memory-Based Thamslation". In Proceedings of COLING-90, pages $1.46-152,1990$.

[11] N. Uramoto. "Lexisal and Structural Disambiguation Using an Example-Base". In The 2nd JapanAustralia Joint Symposium on Natural Language Processing, parges 150 160, 1991.

[12] N. Uramoto. "Wxample-Based Word-Sense Disambiguation". MUICF Trunsactions on Information and Systems, F77-D)(2), 1994. 